PALEO

Revue d'archéologie préhistorique

$20 \mid 2008$

Spécial table ronde ( $2^{\mathrm{e}}$ partie) : Le Gravettien : entités régionales d'une paléoculture européenne, Les Eyzies, juillet 2004

\title{
Nouvelles données chronologiques sur l'enfant de la grotte de Fauroux (Lugasson, Gironde)
}

New chronological data on the child of Fauroux cave (Lugasson, Gironde)

Dorothée G. Drucker, Dominique Henry-Gambier et Michel Lenoir

\section{OpenEdition}

Journals

Édition électronique

URL : http://journals.openedition.org/paleo/1669

DOI : 10.4000/paleo.1669

ISSN : $2101-0420$

Éditeur

SAMRA

Édition imprimée

Date de publication : 1 décembre 2008

Pagination : $55-58$

ISSN : $1145-3370$

Référence électronique

Dorothée G. Drucker, Dominique Henry-Gambier et Michel Lenoir, « Nouvelles données chronologiques sur l'enfant de la grotte de Fauroux (Lugasson, Gironde) », PALEO [En ligne], 20 | 2008, mis en ligne le 24 mai 2011, consulté le 07 juillet 2020. URL : http://journals.openedition.org/paleo/1669 ; DOI: https://doi.org/10.4000/paleo.1669

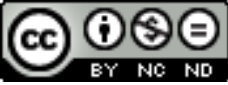

PALEO est mis à disposition selon les termes de la licence Creative Commons Attribution - Pas d'Utilisation Commerciale - Pas de Modification 4.0 International. 


\title{
NOUVELLES DONNÉES CHRONOLOGIQUES SUR L'ENFANT DE LA GROTTE DE FAUROUX (Lugasson, Gironde)
}

\author{
Dorothée G. DRUCKER ${ }^{(1)}$, Dominique HENRY-GAMBIER ${ }^{(2)}$ \\ et Michel LENOIR ${ }^{(3)}$
}

\begin{abstract}
Résumé : La grotte de Fauroux a fourni deux ensembles stratigraphiques attribuables au Magdalénien et à l'Azilien. Des vestiges humains d'un adulte et d'un jeune enfant ont été découverts dans l'un des niveaux de l'Azilien et ont ainsi été attribués à cette culture. Cependant, une confirmation de cette attribution par une datation directe semblait nécessaire au vu des perturbations subies par le site suite à des effondrements et de la présence d'éléments post-paléolithiques. Le fémur gauche de l'individu immature a été retenu et a livré une date de $5035 \pm 30$ ans BP (GrA-38080, $3948-3761$ ans av. J.-C.) qui place finalement ce vestige dans la période du Néolithique.

Mots-clés : collagène, grotte de Fauroux, vestiges humains, datation radiocarbone.

Abstract: New chronological data on the child of Fauroux cave (Lugasson, Gironde). The cave of Fauroux yielded two stratigraphical units attributed to Magdalenian and Azilian culture. Human remains of an adult and a very young child were discovered in association with the Azilian layer and, thus, were attributed to this culture. However, the confirmation of this chronological attributation was needed since the site was disturbed by several collapses and contained post-palaeolithic elements. The left femur from the immature individual was chosen and gave a date of $5035 \pm 30$ years BP (GrA-38080, $3948-3761$ years BC), which places this remain in the Neolithic period.
\end{abstract}

Key-words: collagen, Fauroux cave, human remains, radiocarbon dating.

\section{INTRODUCTION}

La grotte de Fauroux s'ouvre dans un petit vallon affluent de celui du Fontarnaud qui lui-même rejoint la rive droite de l'Engranne, affluent en rive gauche de la basse vallée de la Dordogne. Cette cavité creusée dans le calcaire à Astéries (Rupélien), semble avoir été connue de l'abbé Labrie qui en signala la découverte en 1922, à un kilomètre à l'ouest de la grotte de Fontarnaud, à la faveur d'une carrière souterraine de " la caverne de la Chaume « (La Chaume et Fauroux sont deux lieux-dits proches géographiquement). L'abbé Labrie y entreprit des recherches dont les résultats ne firent l'objet que d'une brève mention sur la présence de Magdalénien (Labrie 1923). La collec- tion Labrie a finalement disparu et le gisement, mentionné ultérieurement par J. Ferrier (Ferrier 1938) et qualifié comme d'importance secondaire, tomba dans l'oubli jusqu'en 1975. A cette date, M. Lenoir et S. Terraza le redécouvrirent en visitant une galerie d'ancienne carrière souterraine que recoupent des petits boyaux naturels très étroits. Ces derniers rejoignent un conduit plus important qui débouche sur un porche d'abri masqué par de gros effondrements et des dépôts récents (Lenoir 1983).

Le remplissage en fond de galerie montrait, sous des apports récents bouleversés par des terriers de renard et de blaireaux, des dépôts plus anciens livrant de l'industrie lithique et de la faune pléistocène. Précédée de sondage,

(1) Universität Tübingen, Institut für Ur- und Frühgeschichte und Archäologie des Mittelarters, Naturwissenschaftliche Archäologie, Rümelinstr. 23, D-72070 Tübingen, Allemagne - dorothee.drucker@ifu.uni-tuebingen.de (2) Université Bordeaux 1, UMR 5199, Laboratoire d'Anthropologie des Populations du Passé, PACEA, avenue des Facultés, 33405 Talence - d.gambier@anthropologie.u-bordeaux1.fr

(3) Université Bordeaux 1, UMR 5199, Institut de Préhistoire et de Géologie du Quaternaire, PACEA, avenue des Facultés, 33405 Talence cedex - m.lenoir@ipgq.u-bordeaux1.fr 
une campagne de fouille fut effectuée en 1978 dans la galerie principale et dans le talus externe en avant de l'effondrement où les dépôts reposaient sur une terrasse rocheuse prolongeant le plancher de l'abri. Dans la galerie principale, ces fouilles ont distingué deux ensembles (I et II). Les niveaux de l'ensemble supérieur (la et lb) ont livré une industrie azilienne (débitage d'éclats et débitage laminaire peu développé, pointes aziliennes). Ceux de l'ensemble inférieur (lla et $\mathrm{llb}$ ) ont permis de mettre en évidence une industrie évoquant davantage le Magdalénien (débitage laminaire, lamelles à dos et burins dièdres). La faune associée aux industries aziliennes comprend surtout des restes de lagomorphes, de Sanglier et de Cerf. Celle des niveaux magdaléniens comporte des vestiges de Renne et de Cheval et témoigne de conditions plus rigoureuses (Gilbert 1984). En outre, dans le talus, une dépression naturelle du plancher rocheux a permis de relever des indices de Solutréen. Des éléments néolithiques et de l'Age du Bronze ont également été identifiés dans des limons brun roux de la partie supérieure des dépôts du talus. Enfin, des limons humiques bruns foncés sus-jacents renfermaient de la céramique médiévale (Lenoir 1983).

Les vestiges d'un adulte et d'un jeune enfant ont été découverts dans un des niveaux aziliens du remplissage à hauteur du porche effondré : niveau lb-carrés E 30, F 30, G 30 (Lenoir 1983 ; Gambier et Lenoir 1991). Toutefois, leur position très superficielle et la faible épaisseur des dépôts paléolithiques ne permettaient pas d'exclure une ancienneté moindre et en particulier une contemporanéité avec les éléments post-paléolithiques représentés dans le talus. II est donc apparu nécessaire de réaliser une datation directe de ces vestiges humains.

\section{Matériel et méthodes}

Les restes humains de la grotte de Fauroux appartiennent à un immature et à un adulte dont l'âge au décès ne peut être précisé. L'enfant est représenté par le tiers distal de l'humérus gauche, le fémur gauche et une deuxième molaire déciduale supérieure droite. La longueur du fémur et ses caractéristiques indiquent un âge au décès se situant entre six et 12 mois (d'après Maresh In Scheuer et Black 2000). La seconde molaire déciduale correspond à un âge dentaire situé entre 12 et 18 mois (Morrees et al.
1963). L'adulte, encore plus fragmentaire, est identifié à partir d'une diaphyse de radius droit privée d'épiphyse distale et d'un premier métatarsien gauche dont les deux épiphyses manquent.

La datation a été effectuée sur le fémur gauche de l'enfant bien conservé (référence de fouille FX-Q1). L'échantillon prélevé sur le milieu de la diaphyse pesait 0,3 gramme (voir tableau). Le collagène a été extrait selon la méthode décrite dans Bocherens et al. (1997) à l'Institut de Préhistoire, Protohistoire et Archéologie du Moyen Âge de Tübingen (Allemagne). Les analyses élémentaires du collagène ( $\mathrm{N}_{\text {coll }}$ et $C_{\text {coll }}$ ont été effectuées au département de Géochimie de l'université de Tübingen (Allemagne). Les rapports $\mathrm{C} / \mathrm{N}$ atomiques du collagène $\left(\mathrm{C} / \mathrm{N}_{\text {coll }}\right)$ permettent de vérifier la fiabilité du résultat de l'analyse isotopique. Ainsi, un $\mathrm{C} / \mathrm{N}_{\text {coll }}$ compris entre 2,9 et 3,6 atteste que les teneurs isotopiques mesurées correspondent bien à celles enregistrées du vivant de l'individu (DeNiro 1985).

\section{Résultat de la datation par carbone 14 et implications chronologiques}

Les mesures AMS effectuées par le Centre de Recherche sur les Isotopes de l'université de Groningen ont permis de dater le collagène extrait de la diaphyse du fémur de 5035 \pm 30 ans BP (GrA-38 080) ce qui correspond à un âge de 3948 - 3761 ans av. J.-C. d'après la courbe de calibration intcal 04 et le logiciel CALIB rev5.0.2@ (Reimer et al. 2004). Le collagène qui a fait l'objet de cette datation présente une composition en carbone et azote qui confirme son intégrité biogéochimique, à savoir un rapport atomique $\mathrm{C} / \mathrm{N}_{\text {coll }}$ compris entre 2,9 et 3,6 et des pourcentages de $\mathrm{N}_{\text {coll }}$ et $\mathrm{C}_{\text {coll }}$ similaires à ceux observés sur des ossements actuels.

Ce résultat exclut ce fossile de la période du Paléolithique supérieur et en particulier de l'Azilien auquel il avait été initialement associé et le renvoie au Néolithique. II est cohérent avec la présence de vestiges néolithiques dans le site.

Les vestiges humains de la grotte de Fauroux constituent un nouvel exemple de vestiges humains récents intrusifs dans un niveau paléolithique et démontrent l'intérêt de datations directes systématiques des vestiges humains.

\begin{tabular}{|c|c|c|c|c|c|c|c|c|}
\hline $\mathbf{N}^{\circ}$ fouille & Échantillon & $\begin{array}{l}\text { Poids os } \\
\text { (g) }\end{array}$ & $\begin{array}{l}\text { rendement coll } \\
(\mathrm{mg} / \mathrm{g})\end{array}$ & $\begin{array}{l}N_{\text {coll }} \\
(\%)\end{array}$ & $\begin{array}{l}\mathrm{C}_{\text {coll }} \\
(\%)\end{array}$ & $\mathrm{C} / \mathrm{N}_{\text {coll }}$ & $\begin{array}{l}\text { Date }{ }^{14} \mathrm{C} \text { BP } \\
\text { (ans) }\end{array}$ & $\begin{array}{l}\text { Date }{ }^{14} \mathrm{C} \text { cal BC, } 2 \sigma \\
\text { (ans) }\end{array}$ \\
\hline FX-Q1 & diaphyse & 0,3 & 122,7 & 43,4 & 15,4 & 3,3 & $5035 \pm 30$ GrA -38080 & 3948- 3761 \\
\hline
\end{tabular}

Tableau - Résultats des analyses élémentaires $\left(N_{\text {coll, }} C_{\text {coll }}\right)$ et de la datation $\left({ }^{14} \mathrm{C}\right)$ réalisées sur le collagène extrait du fémur de l'enfant de Fauroux. La calibration a été effectuée d'après la courbe de calibration intcal 04 et le logiciel CALIB rev5.0.2@.

Table - Results of elementary analysis and datation performed on collagen extracted of Fauroux child's femur. Calibration based on by the curve of calibration intcal04 and the program CALIB rev5.0.2@. 


\section{Remerciements}

Nous remercions les professeurs N.J. Conard et M. Satir de l'université de Tübingen pour nous avoir permis de mener à bien la préparation et l'analyse chimique du collagène.

\section{BIBLIOGRAPHIE}

BOCHERENS H., BILLIOU D., PATOU-MATHIS M., BONJEAN D., OTTE M. et MARIOTTI A. 1997 - Paleobiological implications of the isotopic signature $\left({ }^{13} \mathrm{C},{ }^{15} \mathrm{~N}\right)$ of fossil mammal collagen in Scladina cave (Sclayn, Belgium). Quaternary Research, 48, p. 370-380.

DENIRO M.J. 1985 - Postmortem preservation and alteration of in vivo bone collagen isotope ratios in relation to palaeodietary reconstruction. Nature, 317, p. 806-809.

FERRIER J. 1938 - La Préhistoire en Gironde. Monnoyer imp. Le Mans, 336 p., 31 fig., 85 pl.

GAMBIER D. et LENOIR M. 1991 - Les vestiges humains du Paléolithique supérieur en Gironde. Bulletin de la Société d'Anthropologie du Sud-Ouest, 26 (1), p. 2-31.

GILBERT A. 1984 - Contribution à l'étude des faunes de la fin des temps glaciaires et du début des temps glaciaires. Thèse de l'Université de Bordeaux I, Bordeaux, 1984, 322 p.
LABRIE J. 1923 - Les cavernes et abris préhistoriques de l'Entre-deux-Mers (Gironde). A.F.A.S, 47ème session, Bordeaux 1923, p. 657-663.

LENOIR M. 1983 - Le Paléolithique supérieur des basses vallées de la Dordogne et de la Garonne. Thèse de Doctorat d'Etat es Sciences, Bordeaux : Université de Bordeaux I, 1983. 3t., 702 p., 445 fig., 43 tabl.

MOORREES C.F.A., FANNING E.A. et HUNT E.E. 1963 Formation and Resorption of Three Deciduous Teeth in Children. American Journal of Physical Anthropology, 21, p. 205-213.

REIMER P.J., BAILLIE M.G.L., BARD E., BAYLISS A., BECK J.W., BERTRAND C.J.H., BLACKWELL P.G., BUCK C.E., BURR G.S., CUTLER K.B., DAMON P.E., EDWARDS R.L., FAIRBANKS R.G., FRIEDRICH M., GUILDERSON T.P., HOGG A.G., HUGHEN K.A., KROMER B., MCCORMAC F.G., MANNING S., BRONK RAMSEY C., REIMER R.W., REMMELE R.S., SOUTHON J.R., STUIVER M., TALAMO S., TAYLOR F.W., VAN DER PLICHT J. et WEYHENMEYER C.E. 2004 - INTCAL04 terrestrial radiocarbon age calibration, 0-26 cal kyr BP. Radiocarbon, 46, p. 1029-1058.

SCHEUER L. et BLACK S. 2000 - Developmental Juvenile Osteology. Elsevier Academic Press, Bath, 587 p. 
